\title{
Prevalence and phylogenetic analysis of canine kobuviruses in diarrhoetic dogs in northeast China
}

\author{
Chunqiu $\mathrm{LI}^{1}$, Shan $\mathrm{WEI}^{1}$, Donghua GUO ${ }^{1}$, Zhihui $\mathrm{WANG}^{1)}$, Yufei $\mathrm{GENG}^{1}$, Enyu $\mathrm{WANG}^{1}$, Xiwen $\mathrm{ZHAO}^{1)}$, \\ Mingjun $\mathrm{SU}^{1)}$, Xinyu WANG ${ }^{1)}$ and Dongbo $\mathrm{SUN}^{1) *}$ \\ ${ }^{1)}$ College of Animal Science and Veterinary Medicine, Heilongjiang Bayi Agricultural University, No. 2 Xinyang Road, Sartu District, \\ Daqing 163319, P.R. China
}

(Received 8 July 2015/Accepted 23 July 2015/Published online in J-STAGE 7 August 2015)

ABSTRACT. Canine kobuviruses (CaKVs) are newly recognized picornaviruses that have been recently detected in dogs in the U.S.A., Italy, U.K., the Republic of Korea and Tanzania. To trace the evolution of CaKV strains, a total of 201 fecal samples from rectal swabs of diarrheic dogs, which were obtained from May 2014 to April 2015 in northeast China, were detected by reverse transcription-PCR targeting a partial $(504 \mathrm{bp}$ ) fragment of the 3D gene. Furthermore, a phylogenetic analysis of the CaKV strains identified in northeast China was conducted based on the partial 3D gene sequence. The results indicated that 36 fecal samples $(17.91 \%, 36 / 201)$ were positive for CaKV, in which the co-infection rates of canine coronavirus, canine parvovirus- 2 and canine bocavirus were $58.33 \%, 41.67 \%$, and $11.11 \%$, respectively. Sequence comparison of the partial 3D gene revealed nucleotide homologies of $94.4-100 \%, 95.6-98.6 \%, 94.3-97.6 \%, 94.4-96.3 \%$ and $93.3-95.1 \%$ within the 36 Chinese CaKV strains, and between the 36 Chinese CaKV strains and four CaKV reference strains from South Korea, Italy, U.S.A. and Tanzania, respectively. A phylogenetic tree revealed that the 36 Chinese CaKV strains formed one specific CaKV lineage with CaKVs that have recently been identified in other countries. The 36 Chinese CaKV strains were closely related to CaKV reference strains from Asia and Europe, but differed genetically from CaKV reference strains from North America and Africa. This study provides evidence that CaKVs circulate in diarrhoetic dogs in China and that they exhibit substantial genetic diversity and high co-infection rates with other enteric viruses.

KEY WORDS: canine kobuvirus, epidemiology, genetic diversity, kobuvirus

doi: 10.1292/jvms.15-0414; J. Vet. Med. Sci. 78(1): 7-11, 2016

The family Picornaviridae is highly diverse, currently comprising 17 genera, many of which consist of several species, subspecies and genotypes [1]. Kobuvirus is a newly recognized genus in the family Picornaviridae. Several studies have demonstrated that kobuviruses are transmitted through the fecal-oral route via the consumption of contaminated food or water, and they have a potential role in gastroenteritis in humans and animals $[2,8,11]$. Canine kobuviruses (CaKVs) have been recently discovered in dogs [10], demonstrating that pets can be infected with members of this genus. At present, epidemiological investigations of CaKVs in dogs are important for understanding their pathogenicity and given the interactions between pets and humans, their zoonotic potential.

To date, newly described CaKVs have been detected in healthy and diarrhoetic dogs in the US, Africa, Italy and the U.K. [4, 7, 10, 13, 15, 16]. However, the epidemiology of $\mathrm{CaKVs}$ in China remains unknown. To obtain information regarding the epidemiology of $\mathrm{CaKVs}$, a surveillance study was conducted in diarrhoetic dogs in northeast China from May 2014 to April 2015. Furthermore, the phylogeny of the

*Correspondence to: Sun, D., College of Animal Science and Veterinary Medicine, Heilongjiang Bayi Agricultural University, No. 2 Xinyang Road, Sartu District, Daqing 163319, P.R. China. e-mail: dongbosun@126.com

(C)2016 The Japanese Society of Veterinary Science

This is an open-access article distributed under the terms of the Creative Commons Attribution Non-Commercial No Derivatives (by-nc-nd) License $<$ http://creativecommons.org/licenses/by-nc-nd/3.0/>.
CaKV strains identified in China was analyzed. Our aim was to increase our understanding of the evolution of CaKVs.

\section{MATERIALS AND METHODS}

Sampling: In total, 201 rectal swab samples were collected in diarrhoetic dogs from animal hospitals in three districts-Harbin, Daqing and Mudanjiang — of Heilongjiang province in northeast China from May 2014 to April 2015 using 3.5-m $l$ commercial virus sampling tubes (YOCON Biological Technology Co.. Ltd., Beijing, China). Of the 201 samples, 141 were collected from 2 animal hospitals in Harbin, 20 were collected from one animal hospital in Daqing, and 40 were collected from 1 animal hospital in Mudanjiang. All rectal swab samples were stored at $-80^{\circ} \mathrm{C}$.

$R N A$ and DNA extraction: After $1 \mathrm{ml}$ of fecal samples was centrifuged at $1,500 \times g$ for $10 \mathrm{~min}$ at $4^{\circ} \mathrm{C}$, the supernatant of each sample was transferred to a $1.5-\mathrm{m} l$ Eppendorf (EP) tube. Viral RNA was extracted from each sample using the TIANamp Virus RNA Kit (Tiangen Biotech Co., Ltd., Beijing, China), according to the manufacturer's instructions. Genomic DNA was extracted from the supernatant using a commercial TIANamp Stool DNA Kit (Tiangen Biotech Co., Ltd.), according to the manufacturer's instructions. The extracted RNA and genomic DNAs were stored at $-80^{\circ} \mathrm{C}$.

Detection and sequence analysis of CaKVs: Molecular detection of CaKVs was conducted using reverse transcriptionPCR (RT-PCR) targeting a 504-bp fragment of the 3D gene of CaKV described by Di Martino et al. [7]. The first cDNA was synthesized using random primers (6 nt) by Moloney 
Table 1. Characteristics of the CaKV positive dogs in northeast China and co-infection of CaKVs with other enteric viruses

\begin{tabular}{|c|c|c|c|c|c|c|c|c|c|}
\hline No. & Strain & $\begin{array}{l}\text { Accession } \\
\text { No. }\end{array}$ & $\begin{array}{c}\text { Collection } \\
\text { date }\end{array}$ & Location & Breed & Gender & Age & Vaccined & Other enteric viruses \\
\hline 1 & MDJ-10 & KT210390 & Nov-2014 & MDJ & MI & M & $4 \mathrm{M}$ & NO & CCoV (KT192642) \\
\hline 2 & MDJ-13 & KT210391 & Oct-2014 & MDJ & LR & $\mathrm{F}$ & $2.5 \mathrm{M}$ & NA & CBoV (KR998481) \\
\hline 3 & MDJ-27 & KT210392 & Apr-2015 & MDJ & NA & $\mathrm{F}$ & NA & NA & CBoV (KR998487), CPV-2 (KT074266) \\
\hline 4 & MDJ-38 & KT210393 & Feb-2015 & MDJ & $\mathrm{BF}$ & M & $3.5 \mathrm{M}$ & Yes & - \\
\hline 5 & HRB-a1 & KT210394 & Sep-2014 & HRB & NA & $\mathrm{F}$ & $3.5 \mathrm{M}$ & Yes & $\begin{array}{l}\text { CBoV (KR998488), CCoV (KT192656), } \\
\text { CPV-2 (KT074276) }\end{array}$ \\
\hline 6 & HRB-a3 & KT210395 & Sep-2014 & HRB & GM & M & NA & No & $\operatorname{CCoV}(\mathrm{KT} 192658)$ \\
\hline 7 & HRB-A4 & KT210396 & Sep-2014 & HRB & NA & $\mathrm{F}$ & $3 \mathrm{M}$ & NA & CCoV (KT192652), CPV-2 (KT074319) \\
\hline 8 & HRB-A7 & KT210397 & Sep-2014 & HRB & $\mathrm{SN}$ & M & $3 \mathrm{M}$ & No & $\operatorname{CCoV}(\mathrm{KT} 192654)$ \\
\hline 9 & HRB-A8 & KT210398 & Sep-2014 & $\mathrm{HRB}$ & NA & $\mathrm{F}$ & $1.5 \mathrm{M}$ & Yes & CCoV (KT192655), CPV-2 (KT074280) \\
\hline 10 & DQ-alpha3 & KT210399 & Jan-2015 & DQ & $\mathrm{CH}$ & M & $2 \mathrm{M}$ & No & $\operatorname{CCoV}(\mathrm{KT} 192661)$ \\
\hline 11 & DQ-alpha5 & KT210400 & Feb-2015 & DQ & $\mathrm{PD}$ & NA & $3 \mathrm{M}$ & NA & CCoV (KT192662) \\
\hline 12 & DQ-alpha9 & KT210401 & Feb-2015 & DQ & $\mathrm{CH}$ & M & $1.5 \mathrm{M}$ & Yes & CBoV (KR998489) \\
\hline 13 & DQ-beta3 & KT210402 & Nov-2014 & DQ & NA & $\mathrm{F}$ & $3 \mathrm{M}$ & No & $\operatorname{CCoV}(\mathrm{KT} 192675)$ \\
\hline 14 & HRB-B1 & KT210403 & Sep-2014 & HRB & NA & M & NA & Yes & $\operatorname{CCoV}(\mathrm{KT} 192666)$ \\
\hline 15 & HRB-B0 & KT210404 & Oct-2014 & HRB & PM & $\mathrm{F}$ & $3 \mathrm{M}$ & Yes & CCoV (KT192665), CPV-2 (KT074279) \\
\hline 16 & HRB-B3 & KT210405 & Sep-2014 & HRB & $\mathrm{PD}$ & M & $3 \mathrm{M}$ & Yes & $\operatorname{CCoV}(\mathrm{KT} 192667)$ \\
\hline 17 & HRB-b8 & KT210406 & Oct-2014 & HRB & SM & $\mathrm{F}$ & $2 \mathrm{M}$ & No & CCoV (KT192672), CPV-2 (KT074284) \\
\hline 18 & HRB-B9 & KT210407 & Oct-2014 & HRB & NA & $\mathrm{F}$ & $3 \mathrm{M}$ & Yes & $\operatorname{CCoV}(\mathrm{KT} 192669)$ \\
\hline 19 & HRB-bb1 & KT210408 & Oct-2014 & HRB & NA & $\mathrm{M}$ & $3 \mathrm{M}$ & Yes & - \\
\hline 20 & HRB-bb3 & KT210409 & Oct-2014 & HRB & PM & NA & $2 \mathrm{M}$ & No & CCoV (KT192670), CPV-2 (KT074341) \\
\hline 21 & HRB-C2 & KT210410 & Oct-2014 & HRB & TM & $\mathrm{F}$ & NA & No & CCoV (KT192678), CPV-2 (KT074285) \\
\hline 22 & HRB-D1 & KT210411 & Oct-2014 & HRB & $\mathrm{SN}$ & M & $4 \mathrm{M}$ & Yes & $\operatorname{CCoV}(\mathrm{KT} 192682)$ \\
\hline 23 & HRB-d4 & KT210412 & Nov-2014 & HRB & $\mathrm{CO}$ & $\mathrm{F}$ & $3 \mathrm{M}$ & Yes & - \\
\hline 24 & HRB-D6 & KT210413 & Oct-2014 & HRB & GM & NA & NA & Yes & CCoV (KT192685) \\
\hline 25 & HRB-D9 & KT210414 & Nov-2014 & HRB & NA & NA & $3.5 \mathrm{M}$ & Yes & CPV-2 (KT074296) \\
\hline 26 & HRB-E8 & KT210415 & Nov-2014 & HRB & GM & M & $3 \mathrm{M}$ & Yes & CCoV (KT192687), CPV-2 (KT074300) \\
\hline 27 & HRB-ee8 & KT210416 & Nov-2014 & HRB & $\mathrm{BF}$ & NA & NA & Yes & - \\
\hline 28 & HRB-F1 & KT210417 & Nov-2014 & HRB & $\mathrm{BF}$ & $\mathrm{M}$ & $4 \mathrm{M}$ & Yes & - \\
\hline 29 & HRB-F8 & KT210418 & Nov-2014 & HRB & $\mathrm{PD}$ & M & $3 \mathrm{M}$ & No & CCoV (KT192691), CPV-2 (KT074303) \\
\hline 30 & HRB-G2 & KT210419 & Feb-2015 & HRB & $\mathrm{SN}$ & NA & $4 \mathrm{M}$ & No & - \\
\hline 31 & HRB-G5 & KT210420 & Nov-2014 & HRB & NA & M & $4 \mathrm{M}$ & No & - \\
\hline 32 & HRB-G8 & KT210421 & Nov-2014 & HRB & PM & NA & $4 \mathrm{M}$ & Yes & CPV-2 (KT074346) \\
\hline 33 & HRB-G9 & KT210422 & Feb-2015 & HRB & NA & $\mathrm{F}$ & $3.5 \mathrm{M}$ & No & - \\
\hline 34 & HRB-H2 & KT210423 & Dec-2014 & HRB & $\mathrm{PD}$ & $\mathrm{F}$ & $3 \mathrm{M}$ & NA & CPV-2 (KT074307) \\
\hline 35 & HRB-H6 & KT210424 & Dec-2014 & HRB & PM & NA & $1.5 \mathrm{M}$ & NA & CCoV (KT192695) \\
\hline 36 & HRB-K3 & KT210425 & Jan-2015 & HRB & NA & $\mathrm{F}$ & $3 \mathrm{M}$ & Yes & CPV-2 (KT074317) \\
\hline
\end{tabular}

Note. For breed, GM: Golden Malinois, LR: Labrador Retriever, SM: Samoyed, PM: Pomeranian, SN: Schnauzer, BF: Bijon Frise, CO: Caucasian Owtcharka, TM: Tibetan Mastiff, PD: Poodle, CH: Chihuahua and MI: Manifold Intelligence; For gender, F: female and M: male; for age, M: month; for location, MDJ: Mudanjiang, HRB: Harbin, DQ: Daqing.

murine leukemia virus (RNase H-) reverse transcriptase (Novoprotein Scientific Inc., Shanghai, China), according to the manufacturer's instructions. PCR amplifications were conducted in a $50-\mu l$ reaction volume containing $0.1 \mu \mathrm{M}$ of the forward primer, $0.1 \mu \mathrm{M}$ of the reverse primer, $4 \mu l$ of cDNA, $25 \mu l$ of EmeraldAmp PCR Master Mix (2× Premix) (Takara Biotechnology Co., Ltd., Dalian, China) and an appropriate volume of double-distilled water. The mixtures were amplified by 35 cycles of $94^{\circ} \mathrm{C}$ for $45 \mathrm{sec}, 54^{\circ} \mathrm{C}$ for $1 \mathrm{~min}$ and $72^{\circ} \mathrm{C}$ for $50 \mathrm{sec}$, followed by a final extension at $72^{\circ} \mathrm{C}$ for $10 \mathrm{~min}$ in an Applied Biosystems GeneAmp PCR System 9700 thermal cycler (Thermo Fisher Scientific, Waltham, MA, U.S.A.). After the amplification products were purified using the AxyPrep DNA Gel Extraction kit
(Corning, Suzhou, China), the purified products were directly subjected to sequencing using the Sanger sequencing method. Each sample was sequenced three times. Thirty-six sequences were submitted to GenBank at the National Center for Biotechnology Information (http://www.ncbi.nlm.nih. gov) under the accession numbers KT210390-KT210425.

Phylogenetic analysis of the $3 D$ gene: For the phylogenetic analysis, partial sequences of the 3D gene of kobuviruses from canine and other species were retrieved from GenBank. To construct the phylogenetic trees, a multiple alignment of all target sequences was performed using ClustalX program version 1.83 [18]. Furthermore, phylogenetic trees of all target sequences were generated from the ClustalX-generated alignments by MEGA6.06 software using the neighbor-join- 
ing method [17]. Neighbor-joining phylogenetic trees were built with the p-distance model, 1,000 bootstrap replicates and, otherwise, the default parameters in MEGA 6.

Screening for canine enteric pathogens: All fecal samples that tested positive for $\mathrm{CaKV}$ were also screened for canine parvovirus type 2 (CPV-2), canine coronavirus (CCoV), canine astrovirus (CaAstV), canine norovirus $(\mathrm{CNoV})$, canine bocavirus (CBoV) and Group A-Rotavirus (RV-A) by either PCR or RT-PCR, followed by sequencing, according to previously described protocols $[3,5,6,9,12,14]$.

\section{RESULTS}

Detection of CaKV: The characteristics of the CaKVpositive dogs and co-infections with other enteric viruses are shown in Table 1, and further analysis of the CaKV-positive samples is shown in Table 2. Of the 201 fecal samples, $17.91 \%$ (36/201) were positive for $\mathrm{CaKV}$, and the CaKVpositive rates in Harbin, Daqing and Mudanjiang were $19.86 \%$ (28/141), 20\% (4/20) and 10\% (4/40), respectively. Co-infections with $\mathrm{CCoV}, \mathrm{CPV}-2$ and $\mathrm{CBoV}$ were found in the $36 \mathrm{CaKV}$-positive samples; 58.33\% (21/36) were positive for CCoV; $41.67 \%$ (15/36) were positive for CPV-2; $11.11 \%$ (4/36) were positive for $\mathrm{CBoV} ; 27.78 \%$ (10/36) were positive for $\mathrm{CCoV}$ and $\mathrm{CPV}-2 ; 5.56 \%(2 / 36)$ were positive for CPV-2 and CBoV; and $2.78 \%(1 / 36)$ were positive for $\mathrm{CCoV}, \mathrm{CPV}-2$ and $\mathrm{CBoV}$.

Sequence homology and phylogeny analyses of CaKV strains: Sequence comparisons of the partial 3D gene revealed 94.4-100\%, 95.6-98.6\%, 94.3-97.6\%, 94.4-96.3\%, $93.3-95.1 \%, 84.7-85.4 \%, 81.7-84 \%, 79.6-80.6 \%, 74.3-$ $75.9 \%, 70.6-71.8 \%, 67.4-69 \%$ and $70.1-71.3 \%$ nucleotide identities within the 36 Chinese CaKV strains, between the 36 Chinese CaKV strains and four CaKV reference strains from South Korea, Italy, the USA and Tanzania, respectively, and between the 36 Chinese CaKV strains and seven kobuvirus reference strains from cats, mice, humans (Aichi virus), birds, goats, pigs and bovines, respectively (Table 3 ). Furthermore, the phylogenetic tree revealed that the 36 Chinese CaKV strains formed one specific CaKV lineage with the CaKVs that were recently identified in the other countries; the CaKV lineage was composed of three groups, GI, GII and GIII; one Chinese strain, HRB-A4, belonged to the GII group, while the other 35 Chinese strains belonged to the GI group, which was composed of three subgroups, GIa, GIb and GIc (Fig. 1).

\section{DISCUSSION}

Kobuvirus is a relatively new and emerging genus of the $P i$ cornaviridae, with members infecting both humans and other animal species. In the current study, we conducted prevalence and phylogenetic analyses of canine kobuviruses in diarrhoetic dogs in northeast China. In our study, 36 of 201 (17.91\%) fecal samples from diarrhoetic dogs were positive for $\mathrm{CaKV}$, and the CaKV-positive rates in Harbin, Daqing and Mudanjiang were 19.86\% (28/141), 20\% (4/20) and 10\% (4/40), respectively. In a previous study, a low prevalence of CaKV was reported by RT-PCR in the U.S.A. and Italy. Li et al. [13] reported that

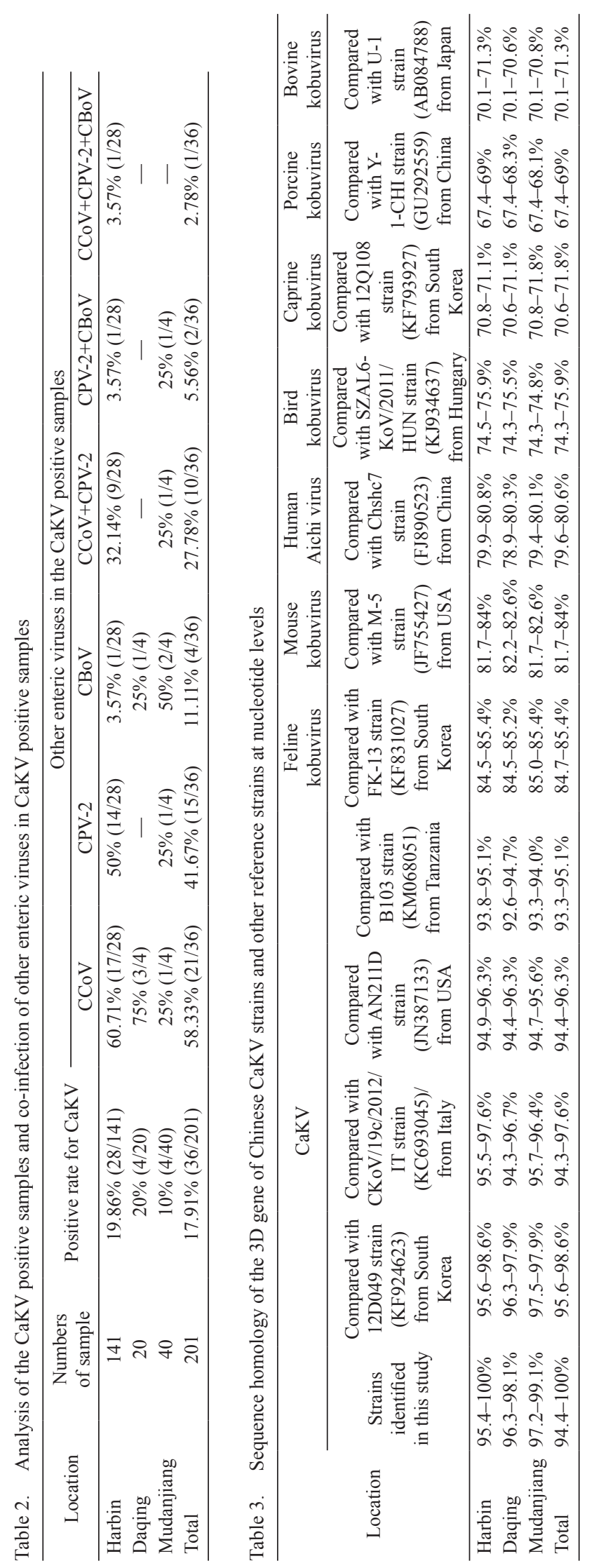




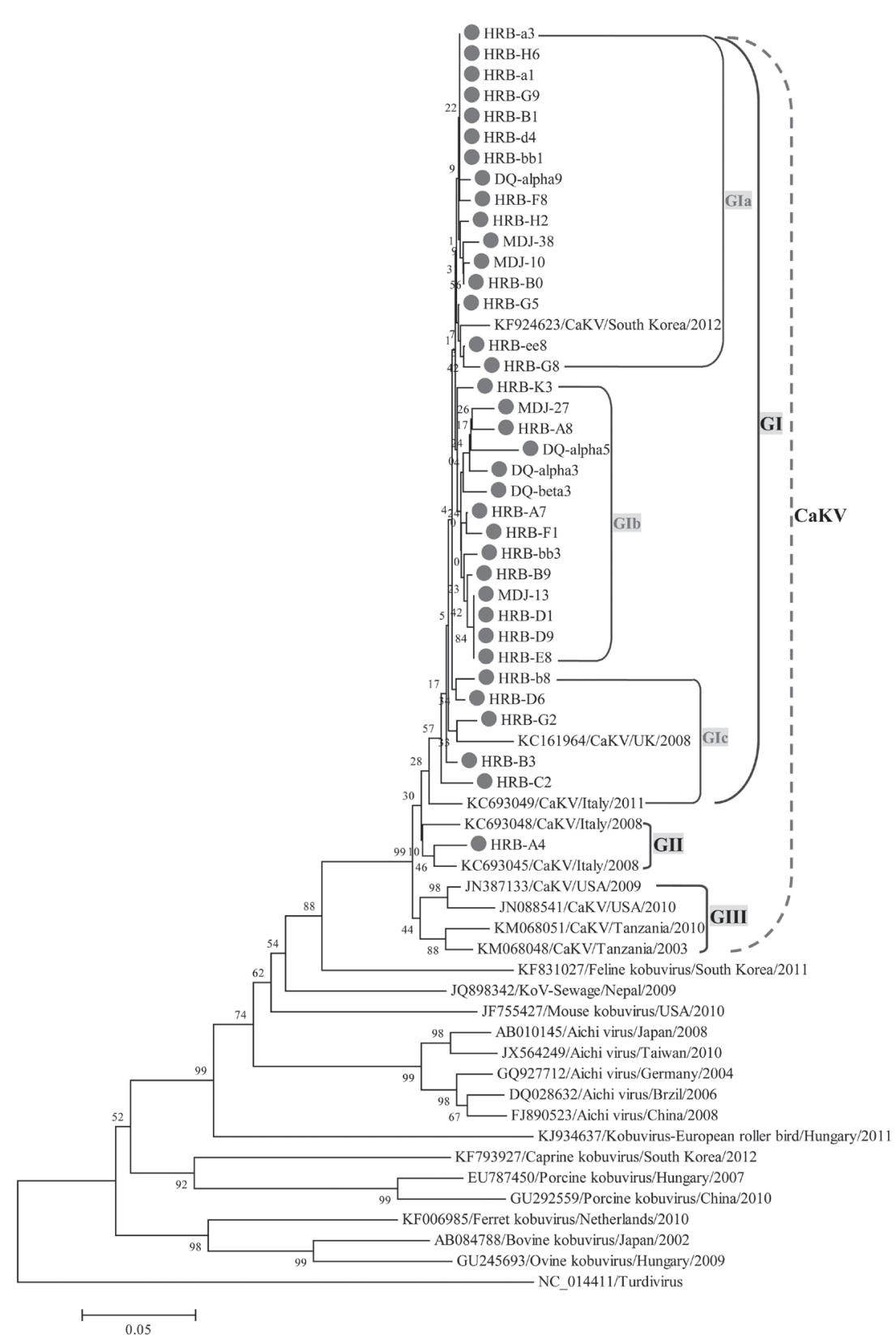

Fig. 1. Phylogenetic analysis of the CaKVs on the basis of the partial 3D gene. •represents the CaKV strains identified in this study.

$10 \%(20 / 200)$ of both sick and healthy dogs tested positive for CaKV. Di Martino et al. [7] reported that 4.37\% (6/137) of diarrhoetic dogs tested positive for CaKV. In contrast, a high prevalence of $\mathrm{CaKV}$ was reported in recent studies from the Republic of Korea. Oem et al. [15] reported that $19.04 \%$ $(4 / 21)$ of diarrhoetic dogs tested positive for CaKV, while Choi et al. [5] reported that 50.55\% (46/91) of diarrhoetic dogs tested positive for CaKV. The $17.91 \% \mathrm{CaKV}$-positive rate in our study is in agreement with that described by Oem et al. [15]. These data suggest that the prevalence of CaKVs may differ among different countries or regions.
These viruses have been implicated in diarrheal disease, but the evidence for a primary pathogenic role remains controversial. In our study, the CaKV-positive fecal samples were also screened for the leading causes of canine viral enteritis, including CPV-2, CCoV, CaAstV, CNoV, CaKV and RV-A. Co-infections with CPV-2, CCoV and $\mathrm{CBoV}$ were identified in the $36 \mathrm{CaKV}$-positive samples. Co-infection of $\mathrm{CaKV}$ with $\mathrm{CCoV}$ reached $58.33 \%(21 / 36)$, and co-infection of CaKV with CPV-2 reached $41.67 \%$ (15/36). These results are similar to those reported in a previous study of $\mathrm{CaKV}$ positive samples from diarrhoetic dogs, in which the co-infec- 
tion rates with CPV-2 and CCoV were 24.81\% (34/137) and $22.62 \%$ (31/137), respectively [7]. In our study, CaKV was the only enteric virus identified in eight specimens, accounting for $22.22 \%(8 / 36)$ of all CaKV-positive samples. This result implies that $\mathrm{CaKV}$ alone may cause infections in the general population. However, the high co-infection rate of $\mathrm{CaKV}$ with other enteric viruses should be considered to be a composite factor for the occurrence of viral diarrhea in dogs in northeast China.

To investigate the genetic heterogeneity of CaKVs circulating in dogs in northeast China, sequence comparison and phylogeny analyses of the $36 \mathrm{CaKV}$-positive strains were conducted on the basis of the partial 3D gene sequence. Genetic analysis of the partial 3D region showed that the CaKVs identified in the Republic of Korea were more variable, sharing nucleotide sequence identities ranging from $85.1 \%$ to $99.8 \%$ [15]. The $36 \mathrm{CaKV}$-positive strains identified in China shared nucleotide sequence identities ranging from $94.4 \%$ to $100 \%$ and, thus, exhibited limited genetic diversity. The phylogenetic tree derived from the partial 3D gene from human and various animal species showed that CaKVs clustered into a single lineage. This finding supports the view that CaKVs from different countries are not restricted geographically and form a single lineage when compared with kobuviruses from other species. However, the lineage of the CaKV strains formed three groups-GI, GII and GIII - and the 36 Chinese CaKV strains were divided into the GI and GII groups. The 35 Chinese CaKV strains in the GI group were divided into the GIa, GIb and GIc subgroups. All 36 Chinese CaKV strains were closely related to the CaKV reference strains from Asia and Europe, and they differed genetically from the CaKV reference strains from North America and Africa. Thus, our data suggest that the lineages of CaKV strains may exhibit geographically differences. This conclusion needs to be validated by extensive epidemiological surveying in future studies.

In conclusion, the present study reports, for the first time, the prevalence and phylogenetic analysis of canine kobuviruses in diarrhoetic dogs in northeast China, and the CaKV strains circulating in northeast China exhibit genetic diversity and high co-infection rates with other enteric viruses. However, further investigations are needed to determine the relationship between CaKV infections and the ability of CaKVs to cause diarrhea in dogs.

ACKNOWLEDGMENT. This work was supported by the Program for New Century Excellent Talents in Heilongjiang Provincial University (grant no. 1252-NCET-016).

\section{REFERENCES}

1. Adams, M. J., King, A. M. and Carstens, E. B. 2013. Ratification vote on taxonomic proposals to the International Committee on Taxonomy of Viruses (2013). Arch. Virol. 158: 2023-2030. [Medline] [CrossRef]

2. Ambert-Balay, K., Lorrot, M., Bon, F., Giraudon, H., Kaplon, J., Wolfer, M., Lebon, P., Gendrel, D. and Pothier, P. 2008. Prevalence and genetic diversity of Aichi virus strains in stool samples from community and hospitalized patients. J. Clin. Microbiol. 46: 1252-1258. [Medline] [CrossRef]

3. Buonavoglia, D., Cavalli, A., Pratelli, A., Martella, V., Greco, G., Tempesta, M. and Buonavoglia, C. 2000. Antigenic analysis of canine parvovirus strains isolated in Italy. New Microbiol. 23: 93-96. [Medline]

4. Carmona-Vicente, N., Buesa, J., Brown, P. A., Merga, J. Y., Darby, A. C., Stavisky, J., Sadler, L., Gaskell, R. M., Dawson, S. and Radford, A. D. 2013. Phylogeny and prevalence of kobuviruses in dogs and cats in the UK. Vet. Microbiol. 164: 246-252. [Medline] [CrossRef]

5. Choi, S., Lim, S. I., Kim, Y. K., Cho, Y. Y., Song, J. Y. and An, D. J. 2014. Phylogenetic analysis of astrovirus and kobuvirus in Korean dogs. J. Vet. Med. Sci. 76: 1141-1145. [Medline] [CrossRef]

6. Costa, E. M., de Castro, T. X., Bottino Fde, O. and Garcia Rde, C. 2014. Molecular characterization of canine coronavirus strains circulating in Brazil. Vet. Microbiol. 168: 8-15. [Medline] [CrossRef]

7. Di Martino, B., Di Felice, E., Ceci, C., Di Profio, F. and Marsilio, F. 2013. Canine kobuviruses in diarrhoeic dogs in Italy. Vet. Microbiol. 166: 246-249. [Medline] [CrossRef]

8. Drexler, J. F., Baumgarte, S., de Souza Luna, L. K., EschbachBludau, M., Lukashev, A. N. and Drosten, C. 2011. Aichi virus shedding in high concentrations in patients with acute diarrhea. Emerg. Infect. Dis. 17: 1544-1548. [Medline]

9. Gómez, M. M., de Mendonça, M. C., Volotão Ede, M., Tort, L. F., da Silva, M. F., Cristina, J. and Leite, J. P. 2011. Rotavirus A genotype $\mathrm{P}[4] \mathrm{G} 2$ : genetic diversity and reassortment events among strains circulating in Brazil between 2005 and 2009. J. Med. Virol. 83: 1093-1106. [Medline] [CrossRef]

10. Kapoor, A., Simmonds, P., Dubovi, E. J., Qaisar, N., Henriquez, J. A., Medina, J., Shields, S. and Lipkin, W. I. 2011. Characterization of a canine homolog of human Aichivirus. J. Virol. 85: 11520-11525. [Medline] [CrossRef]

11. Khamrin, P., Maneekarn, N., Okitsu, S. and Ushijima, H. 2014. Epidemiology of human and animal kobuviruses. Virusdisease 25: 195-200. [Medline] [CrossRef]

12. Lau, S. K., Woo, P. C., Yeung, H. C., Teng, J. L., Wu, Y., Bai, R., Fan, R. Y., Chan, K. H. and Yuen, K. Y. 2012. Identification and characterization of bocaviruses in cats and dogs reveals a novel feline bocavirus and a novel genetic group of canine bocavirus. J. Gen. Virol. 93: 1573-1582. [Medline] [CrossRef]

13. Li, L., Pesavento, P. A., Shan, T., Leutenegger, C. M., Wang, C. and Delwart, E. 2011. Viruses in diarrhoeic dogs include novel kobuviruses and sapoviruses. J. Gen. Virol. 92: 2534-2541. [Medline] [CrossRef]

14. Mesquita, J. R. and Nascimento, M. S. 2012. Molecular epidemiology of canine norovirus in dogs from Portugal, 2007-2011. BMC Vet. Res. 8: 107. [Medline] [CrossRef]

15. Oem, J. K., Choi, J. W., Lee, M. H., Lee, K. K. and Choi, K. S. 2014. Canine kobuvirus infections in Korean dogs. Arch. Virol. 159: 2751-2755. [Medline] [CrossRef]

16. Olarte-Castillo, X. A., Heeger, F., Mazzoni, C. J., Greenwood, A. D., Fyumagwa, R., Moehlman, P. D., Hofer, H. and East, M. L. 2015. Molecular characterization of canine kobuvirus in wild carnivores and the domestic dog in Africa. Virology 477: 89-97. [Medline] [CrossRef]

17. Tamura, K., Stecher, G., Peterson, D., Filipski, A. and Kumar, S. 2013. MEGA6: Molecular evolutionary genetics analysis version 6.0. Mol. Biol. Evol. 30: 2725-2729. [Medline] [CrossRef]

18. Thompson, J. D., Gibson, T. J., Plewniak, F., Jeanmougin, F. and Higgins, D. G. 1997. The ClustalX windows interface: flexible strategies for multiple sequence alignment aided by quality analysis tools. Nucleic Acids Res. 25: 4876-4882. [Medline] [CrossRef] 